\title{
QT dispersion is reduced after valve replacement in patients with aortic stenosis
}

\author{
D Darbar, C J Cherry, D M Kerins
}

\begin{abstract}
Objective-To investigate whether QT dispersion is a reliable index of the severity of aortic stenosis and left ventricular hypertrophy in the setting of aortic stenosis.

Design-A retrospective analysis of the results of echocardiography and electrocardiography before and after aortic valve replacement.

Setting-Tertiary centre.
\end{abstract}

Patients-36 men (30 white and six black) with symptomatic aortic stenosis requiring valve replacement.

Results-All patients had significant aortic stenosis (mean (SD) aortic valve area $0.68(0.18) \mathrm{cm}^{2}$ ) and evidence of left ventricular hypertrophy (left ventricular mass index (LVMI): 267 (90) $\mathrm{g} / \mathrm{m}^{2}$ ). Before aortic valve replacement, $Q T$ dispersion was correlated with mean aortic valve area and LVMI $(r=0.697, p<0.001$, and $r=0.59, \mathrm{p}<2.4 \times 10^{-6}$, respectively). QT dispersion and QT corrected for heart rate dispersion decreased from 133 (54) to 71 (33) $\mathrm{ms}$ and from 151 (64) to $94(76) \mathrm{ms}$, respectively ( $p<0.001$ for both). LVMI regressed after aortic valve replacement to $190(79) \mathrm{g} / \mathrm{m}^{2}, \mathrm{p}<0.01$.

Conclusions-QT dispersion is increased in association with LVMI in patients with significant symptomatic aortic stenosis. Aortic valve replacement reduces QT dispersion and LVMI. QT dispersion could be a useful indicator of risk and risk reduction in patients with significant symptomatic aortic stenosis.

(Heart 1999;82:15-18)

Keywords: QT dispersion; left ventricular hypertrophy; aortic valve replacement

Department of

Medicine, Vanderbilt

University and

Veterans

Administration

Medical Center,

Nashville, Tennessee,

USA

D Darbar

C J Cherry

D M Kerins

Correspondence to:

Dr David M Kerins, Division of Cardiology, Medical

Research Building-II, Room

315, Vanderbilt University,

Nashville, TN 37232-6300,

USA.

email: david.kerins@mcmail.

vanderbilt.edu

Accepted for publication 22 December 1998 stenosis. ${ }^{6}$ It most often occurs in subjects with a history of heart failure, but may also be seen in asymptomatic individuals $\left(3-5 \%\right.$ in adults $\left.{ }^{6}\right)$. Although the factors predisposing patients with symptomatic aortic stenosis to an increased risk of sudden cardiac death are not fully known, the presence of left ventricular hypertrophy may be one contributing factor, since it is often encountered in this group of patients. ${ }^{9}$

A simple variable currently under investigation in cardiac disease is QT dispersion. ${ }^{10} \mathrm{With}$ this technique, the QT interval is calculated for all 12 ECG leads, and the shortest interval is subtracted from the longest interval to give the QT dispersion. An increased QT dispersion reflects regional variation in ventricular repolarisation in the heart, which may predispose to reentrant arrhythmias. It has been shown to be predictive of cardiac death in patients with a broad spectrum of disease. ${ }^{10-14}$ In this study, we investigated the relation between QT dispersion and left ventricular hypertrophy in a group of patients with significant aortic stenosis who underwent aortic valve replacement. Our hypothesis was that patients with significant aortic stenosis and left ventricular hypertrophy would have increased QT dispersion before aortic valve replacement and that QT dispersion would be reduced in association with regression of left ventricular hypertrophy after valve replacement.

\section{Methods}

The medical records in the cardiology section of the university affiliated Veterans Administration Medical Center from 1984 to 1996 were reviewed retrospectively for patients with an established diagnosis of aortic stenosis who underwent aortic valve replacement during this period. Inclusion criteria were the availability of 12 lead ECGs and two echocardiograms of good quality, one performed before aortic valve replacement and a second one a minimum of 12 months after valve surgery.

Exclusion criteria included a history of previous myocardial infarction (by history or the presence of pathological Q waves on ECG), angiographically significant coronary artery disease ( $>50 \%$ stenosis in one of the main epicardial coronary arteries), sustained ventricular tachycardia, moderate to severe aortic incompetence by Doppler echocardiography, or a left ventricular ejection fraction of $<45 \%$. Subjects whose 12 lead ECGs were of poor quality, had bundle branch block, atrial fibrillation, or were taken while the patient was on an antiarrhythmic agent known to prolong the QT interval were also excluded, as were patients who were on angiotensin converting enzyme valve replacement. ${ }^{6-8}$ Complex ventricular arrhythmias are significantly more prevalent in aortic stenosis than in control subjects, and sudden cardiac death accounts for about one third of the mortality of unoperated aortic 
Table 1 Echocardiographic and QT measurements before and after aortic valve replacement

\begin{tabular}{lll}
\hline & Baseline & Follow up \\
\hline Heart rate (beats/min) & $69(8.2)$ & $66(10)$ \\
LVID $(\mathrm{cm})$ & $5.33(0.68)$ & $4.99(0.62)$ \\
IVS $(\mathrm{cm})$ & $1.35(0.26)$ & $1.16(0.25)$ \\
PWT $(\mathrm{cm})$ & $1.26(0.19)$ & $1.14(0.11)$ \\
LVMI $\left(\mathrm{g} / \mathrm{m}^{2}\right)$ & $267(90)$ & $190(79)^{\star \star}$ \\
Aortic valve gradient $\left(\mathrm{cm}^{2}\right)$ & $45.2(11)$ & $15.2(6.2)^{\star \star \star}$ \\
Maximum QT $(\mathrm{ms})$ & $476(64)$ & $388(45)^{\star \star}$ \\
Minimum QT $(\mathrm{ms})$ & $346(33)$ & $318(38)$ \\
QT dispersion $(\mathrm{ms})$ & $133(54)$ & $71(33)^{\star \star \star}$ \\
QTc dispersion $(\mathrm{ms})$ & $151(64)$ & $94(76)^{\star \star \star}$
\end{tabular}

Values are mean (SD)

${ }^{\star \star} \mathrm{p}<0.01 ;{ }^{\star \star \star} \mathrm{p}<0.001$

IVS, interventricular septum; LVID, left ventricular internal dimension; LVMI, left ventricular mass index; PWT, posterior wall thickness.

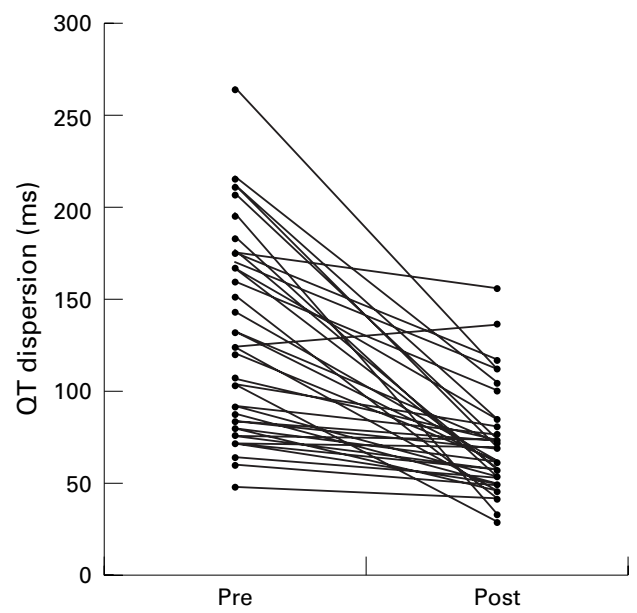

Figure 1 Individual $Q T$ dispersion values before and after aortic valve replacement

(ACE) inhibitor treatment at baseline or at follow up. ${ }^{15}$

All 12 lead ECGs were recorded with the patient resting supine, using an analogue system at $25 \mathrm{~mm} /$ second paper speed, 10 $\mathrm{mm} / \mathrm{mV}$ gain, and $40 \mathrm{~Hz}$ low pass filter setting. The QT intervals were measured on all possible leads by an experienced observer blinded to all clinical details using a semiautomated digitising program. The QT intervals were taken to be from the onset of the QRS to the end of the $\mathrm{T}$ wave by a tangential method. ${ }^{11}$ If $U$ waves were present the QT interval was measured to the nadir of the curve between the $\mathrm{T}$ and $\mathrm{U}$ waves, also with the aid of a tangent. Three consecutive cycles were measured for

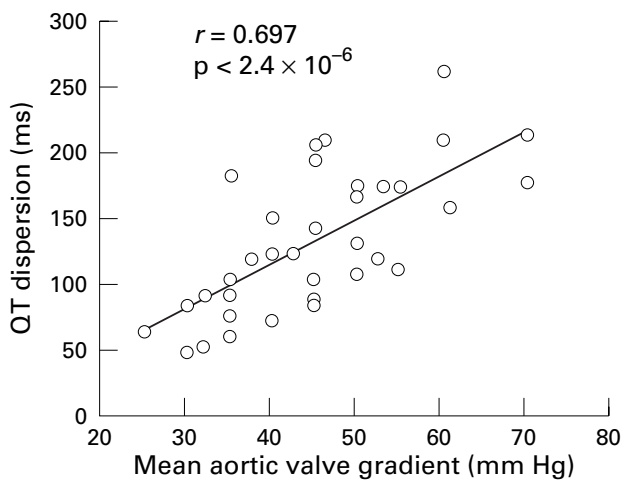

Figure 2 Relation between $Q T$ and mean aortic valve area before aortic valve replacement. each lead. QT intervals were corrected with Bazett's formula $\left(\mathrm{QTc}=\mathrm{QT} / \mathrm{RR}^{1 / 2}\right)$. QT dispersion was determined as the difference between maximum and minimum QTc interval in different leads. No subject had fewer than nine measurable leads.

To determine interobserver variability, a second observer made independent blinded QT determinations of 20 randomly selected ECGs. Intraobserver variability was evaluated by QT analysis of 30 randomly assigned ECGs, again in a blinded fashion by a single observer. Interobserver measurement error was avoided by using the measurements of the same experienced observer for statistical comparisons.

Left ventricular measurements from the $M$ mode echocardiograms were made by two physicians who were blinded to ECG measurements. The echocardiograms were evaluated according to the recommendation of the American Society of Echocardiography. ${ }^{16} \mathrm{Di}-$ astolic measurements of left ventricular interval dimensions, ventricular septal thickness, and posterior wall were obtained. Left ventricular mass was calculated according to the formula: left ventricular mass $=1.04$ [(LVID + PWT + IVST $\left.)^{3}-(\text { LVID })^{3}\right]-13.6 \mathrm{~g},{ }^{17}{ }^{18}$ where LVID is left ventricular internal dimension, PWT is posterior wall thickness, and IVST is interventricular septal dimension. Left ventricular mass index (LVMI) was determined by dividing left ventricular mass by body surface area.

\section{STATISTICS}

All data are expressed as mean (SD). Linear regression analysis was performed between LVMI, mean aortic valve area, and QT dispersion measures. The Student's paired $t$ test was used to compare QT dispersion before and after aortic valve replacement with the chosen statistical significance level of $\mathrm{p}<0.05$.

\section{Results}

The study group consisted of 36 men of whom 30 were white and the remainder black (African-American). The mean (SD) age was 62 (8.1) years, with baseline left ventricular ejection fraction of $58(7.2) \%$. The mean aortic valve area before aortic valve replacement was $0.68(0.18) \mathrm{cm}^{2}$. All patients in the study group had one or more of the following symptoms - chest pain, syncope, or dyspnoea on exertion, which necessitated aortic valve replacement. Nineteen (53\%) of the patients had a history of hypertension, eight $(22 \%)$ had chronic obstructive pulmonary disease, seven $(19 \%)$ had peripheral vascular disease, and three $(8 \%)$ had diabetes mellitus. Echocardiograms and ECGs at baseline were performed 7 (5) and 3 (1) days, respectively, before aortic valve replacement and were then repeated 620 (184) and 580 (230) days after valve replacement. The time interval between follow up echocardiograms and ECGs was 125 (82) days. Twenty two of the patients had metallic aortic valve prostheses (20 St Jude's and two Bjork-Shiley) and the remaining 14 had Carpentier-Edwards prostheses. 


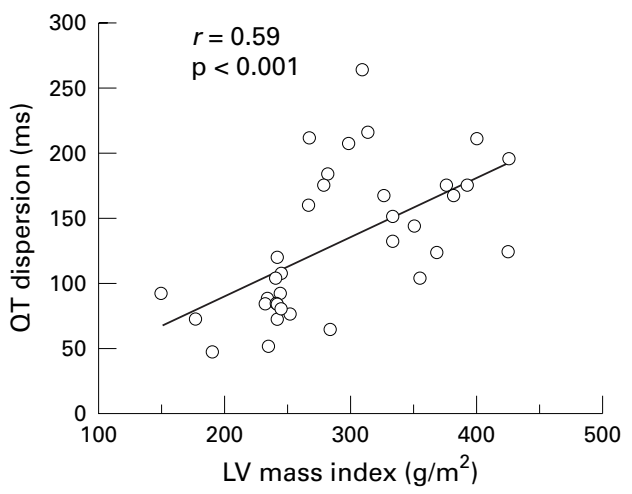

Figure 3 Relation between $Q T$ and left ventricular (LV) mass index before aortic valve replacement.

The echocardiographic and ECG data before and after aortic valve replacement are presented in table 1 . The mean QT dispersion in patients before aortic valve replacement was 133 (54) ms, with a significant reduction after aortic valve replacement (71 (33) ms, $\mathrm{p}<0.001)$. Individual QT dispersion values before and after valve replacement are shown in fig 1. Before aortic valve replacement, QT dispersion was significantly correlated with mean aortic valve gradient (fig 2) and LVMI (fig 3). QTc dispersion was also significantly correlated with mean aortic valve gradient and LVMI $\left(r=0.687, \mathrm{p}<4.0 \times 10^{-6}\right.$ and $r=0.45$, $\mathrm{p}<0.01$, respectively). An important point to note is that all patients in the study satisfied the echocardiographic criteria for left ventricular hypertrophy $\left(\mathrm{LVMI}>131 \mathrm{~g} / \mathrm{m}^{2}\right) .{ }^{19}{ }^{20}$ Intraobserver variability of QT dispersion measurements was $12(6.2) \mathrm{ms}$ with a corresponding interobserver variability of 18 (10) ms.

\section{Discussion}

Our main finding was that patients with significant aortic stenosis and left ventricular hypertrophy have increased QT dispersion, which is reduced after aortic valve replacement. The relation between QT dispersion, LVMI, and aortic valve area suggests that regression of left ventricular hypertrophy after valve replacement may partly be responsible for the significant reduction in QT dispersion.

This study confirms the findings of earlier studies, which have shown that in the absence of significant aortic stenosis, QT dispersion is increased in patients with left ventricular hypertrophy. ${ }^{21-23}$ In particular, QT dispersion had a significant positive correlation with LVMI, indicating inhomogeneity of repolarisation in relation to left ventricular hypertrophy. In previous studies, QT dispersion has been shown to be increased in patients after acute myocardial infarction ${ }^{14}$ and in patients with the long QT syndrome, ${ }^{11}$ chronic heart failure, ${ }^{12}$ and hypertrophic cardiomyopathy. ${ }^{13}$ In these populations, increased QT dispersion has also been shown to be associated with susceptibility to ventricular arrhythmias or sudden cardiac death. ${ }^{11-13}$ Although arrhythmic susceptibility was not assessed in our group of patients, our observations suggest that left ventricular hypertrophy is associated with increased inhomoge- neity in repolarisation, probably predisposing patients with significant aortic stenosis and left ventricular hypertrophy to life threatening arrhythmias.

One possible mechanism responsible for increased QT dispersion in patients with left ventricular hypertrophy may be related to alteration in ion channels responsible for cardiac repolarisation. ${ }^{24-27}$ Abnormalities in the potassium channels in hypertrophied myocytes have been shown to contribute to the prolongation of the action potential. ${ }^{27}$ In addition, studies showing that QT dispersion is longer in hypertensive patients with left ventricular hypertrophy than in those without ${ }^{23}$ suggest that changes in potassium channel physiology owing to hypertrophied myocytes may contribute to increased QT dispersion. Local ischaemia of the hypertrophied ventricle, as well as increased anisotropy and stretching of myocardial fibres, could also be other mechanisms whereby QT dispersion is increased in patients with left ventricular hypertrophy.

Left ventricular hypertrophy is commonly found in patients with symptomatic aortic stenosis $^{9}$ and so may be related to the increased incidence of sudden cardiac death in unoperated patients. Regression of left ventricular hypertrophy after aortic valve replacement could lead to reduced arrhythmic risk. However, it is not possible from this study to comment on whether a reduction in QT dispersion after valve surgery for aortic stenosis would necessarily translate into reduced arrhythmic risk. Studies specifically looking at regression of left ventricular hypertrophy and survival after aortic valve replacement are clearly needed. Nevertheless, QT dispersion could be a useful indicator of risk and risk reduction in patients with significant symptomatic aortic stenosis.

The size of the study population was limited in part by the exclusion of patients with reduced left ventricular systolic function and significant coronary heart disease, since both of these factors can independently influence QT dispersion. ${ }^{28}{ }^{29}$ Clearly, a prospective study in a larger patient sample is needed to define more accurately the precise relation between QT dispersion and regression of left ventricular hypertrophy after aortic valve replacement. Other limitations of the study include variations in the timing of the follow up echocardiograms and ECGs, which were on average performed four months apart; however, these are inherent restrictions of a retrospective study. Although patients on ACE inhibitor treatment were excluded from the study (since this is known to reduce QT dispersion ${ }^{15}$ ), the effect of other drug treatments was not assessed in this study. Therefore, it is possible that these could have biased the results.

Our study has shown that QT dispersion is increased in patients with significant aortic stenosis and left ventricular hypertrophy. Aortic valve replacement leads to a reduction in QT dispersion and regression of left ventricular hypertrophy. QT dispersion could be a useful indicator of risk and risk reduction in patients with significant symptomatic aortic stenosis. 
1 Kannel WB, Dannenberg AL, Levy D. Population implications of electrocardiographic left ventricular hypertrophy. tions of electrocardiographic

2 Levy D, Anderson KM, Savage DD, et al. Risk of ventricular arrhythmias in left ventricular hypertrophy: the Framingham heart study. Am f Cardiol 1987;60:560-5.

3 Levy D, Anderson KM, Plehn J, et al. Echocardiographically determined left ventricular structural and functional correlates of complex or frequent ventricular arrhythmias on one-hour ambulatory electrocardiographic monitoring. $\mathrm{Am}$ f Cardiol 1987;59:836-40.

4 Kannel WB, Doyle JT, McNamara PM, et al. Precursors of sudden coronary death. Factors related to the incidence of sudden death. Circulation 1975;51:606-13.

5 Aguilar J, Martinez A, Conejos F. Mechanisms of ventricular arrhythmias in the presence of pathological hypertrophy. Eur Heart f 1993;14:66-70.

6 Ross J, Braunwald E. Aortic stenosis. Circulation 1968; 38(suppl V):61-7.

7 Schwarz F, Baumann P, Manthey J, et al. The effect of aortic valve replacement on survival. Circulation 1982;66: $1105-10$

8 Schwarz F, Flameng W, Langebartels F, et al. Impaired left ventricular function in chronic aortic valve disease: survival and function after replacement by Bjork-Shiley prosthesis. Circulation 1979;60:48-58.

9 Cohle SD, Graham MA, Dowling G, et al. Sudden death and left ventricular outflow disease. Pathol Ann 1988;23 97-124.

10 Higham PD, Campbell RW. QT dispersion. Br Heart $\mathcal{f}$ 1994;71:508-10.

11 Day CP, McComb JM, Campbell RW. QT dispersion: an indication of arrhythmia risk in patients with long QT intervals. Br Heart f 1990;63:342-4.

12 Barr CS, Naas A, Freeman M, et al. OT dispersion and sudden unexpected death in chronic heart failure. Lancet 1994;343:327-9.

13 Buja G, Miorelli M, Turrini P, et al. Comparison of QT dispersion in hypertrophic cardiomyopathy between patients persion in hypertrophic cardiomyopathy between patients
with and without ventricular arrhythmias and sudden death. Am 7 Cardiol 1993;72:973-6.

14 Glancy JM, Garratt CJ, Woods KL, et al. QT dispersion and mortality after myocardial infarction. Lancet 1995;345. mortali

15 Barr CS, Naas AA, Fenwick M, et al. Enalapril reduces QTc dispersion in mild congestive heart failure secondary to coronary artery disease. Am f Cardiol 1997;79:328-33.
16 Sahn DJ, DeMaria A, Kisslo J, et al. Recommendations regarding quantitation in M-mode echocardiography: results of a survey of echocardiographic measurements. Circulation 1978;58:1072-83.

17 Devereux RB, Reichek N. Echocardiographic determination of left ventricular mass in man. Circulation 1977;55:61318

18 Devereux RB, Alonso DR, Lutas EM, et al. Echocardioraphic assessment of left ventricular hypertrophy: comparison to necropsy findings. Am 7 Cardiol 1986;57:450-8.

19 Houghton JL, Frank MJ, Carr AA, et al. Relations among impaired coronary flow reserve, left ventricular hypertrophy and thallium perfusion defects in hypertensive patients without obstructive coronary artery disease. $7 \mathrm{Am}$ Coll Cardiol 1990;15:43-51.

20 Levy D, Savage DD, Garrison RJ, et al. Echocardiographic criteria for left ventricular hypertrophy: the Framingham Heart Study. Am f Cardiol 1987;59:956-60.

21 Mayet J, Shahi M, McGrath K, et al. Left ventricular hypertrophy and QT dispersion in hypertension. Hypertension 1996;28:791-6.

22 Ichkhan K, Molnar J, Somberg J. Relation of left ventricular mass and QT dispersion in patients with systematic hypertension. Am 7 Cardiol 1997;79:508-11.

23 Perkiomaki JS, Ikaheimo MJ, Pikkujamsa SM, et al. Dispersion of the QT interval and autonomic modulation of heart rate in hypertensive men with and without left ventricular hypertrophy. Hypertension 1996;28:16-21.

24 Rials SJ, Friehling TD, Marinchak RA, et al. Potassium channels in cardiac arrhythmias: focus on antiarrhythmic drug action. Prog Clin Biol Res 1990;334:111-21.

25 Kleiman RB, Houser SR. Calcium currents in normal and hypertrophied isolated feline ventricular myocytes. $A m$ f Physiol 1988;255:H1434-42.

26 Kleiman RB, Houser SR. Outward currents in hypertrophied feline ventricular myocytes. Prog Clin Biol Res 1990; 334:65-83.

$27 \mathrm{McD}$ onald TF, Trautwein W. The potassium current underlying delayed rectification in cat ventricular muscle. $\mathcal{F}$ Physiol (Lond) 1978;274:217-46.

28 Struthers AD, Davidson NC, Naas A, et al. QT dispersion and triple-vessel coronary disease. Lancet 1997;349:11745.

$29 \mathrm{Fu}$ GS, Meissner A, Simon R. Repolarization dispersion and sudden cardiac death in patients with impaired left ventricular function. Eur Heart $\mathcal{f}$ 1997;18:281-9. 